Artículo

\title{
El impacto de la epidemia de gripe de 1918 en La Mancha meridional: estudio de caso
}

\author{
José Cañabate* $\mathbb{D}$ \\ Universidad de Murcia
}

\section{Javier Puche $\mathbb{D}$}

Universidad de Zaragoza. Instituto Agroalimentario de Aragón, IA2 (UNIZAR-CITA)

\section{INFORMACIÓN DEL ARTÍCULO}

\section{Historia del artículo:}

Recibido: 4 de junio de 2020

Aceptado: 22 de febrero de 2021

On-line: 12 de abril de 2021

\section{Códigos JEL}

I10

I12

I18

N34

Palabras clave

Epidemia de gripe

1918

Hellín

La Mancha meridional

\section{R E S U M E N}

Este artículo analiza los efectos que la epidemia de gripe de 1918 tuvo en la morbimortalidad y en la economía en La Mancha meridional. Estudia el municipio de Hellín (Albacete). Los libros de defunciones del Registro Civil, las matrículas de la contribución industrial y de comercio y las actas de Pleno de su Ayuntamiento constituyen las principales fuentes de estudio. Los resultados muestran que, según el índice de Dupâquier, Hellín sufrió una crisis fuerte de mortalidad asociada a la pandemia de gripe, lo que se tradujo en un retroceso de la edad media de supervivencia de su población. La incidencia de la gripe, a diferencia de otros contextos más urbanos, se concentró en otoño de 1918. Se evidencia una leve sobremortalidad masculina, siendo el grupo de edad más afectado el de los 10-39 años. Los efectos de la pandemia en la economía local, por el contrario, fueron más bien leves.

@ 2021 Asociación Española de Historia Económica

\section{JEL classification:}

I10

I12

I18

N34

Keywords:

Influenza epidemic

1918

Hellín

Southern La Mancha
The impact of the 1918 influenza epidemic in southern La Mancha: Case study

\section{A B S T R A C T}

This article analyses the effects that the 1918 influenza epidemic had on the morbidity, the mortality and on the economy in southern La Mancha. This study focuses on the municipality of Hellín (Albacete). The libros de defunciones of the Civil Registry, the matrículas de la contribución industrial y de comercio and the actas de Pleno of the Hellín City Council, constitute the main sources of study. The results show that, according to the Dupâquier Index, Hellín suffered a severe crisis of mortality associated with the influenza pandemic, which was translated into a decrease in the average age of survival of the population of Hellin. The incidence of influenza, unlike other urban contexts, was concentrated in the fall of 1918. A slight excess male mortality has been found, being the most affected the 10-39 year old group. The effects of the pandemic on the local economy, by contrast, were rather mild.

\footnotetext{
* Autor para correspondencia. Correo electrónico: jose.c.c@um.es (José Cañabate). 


\section{Introducción}

Junto al inicio del crecimiento económico moderno y los cambios en la estructura productiva, uno de los hechos más importantes que ha conocido la historia humana desde el siglo xIx ha sido las mejoras en la salud y el aumento de la esperanza de vida de las poblaciones, impulsada en buena medida por los adelantos en la producción alimentaria y en la sanidad pública (Floud et al, 2011, 2014; Pérez Moreda et al., 2015). El aumento del tiempo medio de vida de las poblaciones vino determinado por el proceso de modernización demográfica, un fenómeno propio de los países que han experimentado un progreso de la estructura económica y social (Floud et al, 2011, 2014). Se caracteriza, conjuntamente, por un descenso de las tasas de mortalidad y fecundidad (transición demográfica), y por la reducción de la mortalidad por enfermedades infecciosas (transición epidemiológica) (Pérez Moreda et al., 2015; Sanz Gimeno y Ramiro Fariñas, 2002a).

A finales del siglo xIx, las tasas de mortalidad comenzaron a descender en muchos países europeos (Floud et al, 2011; Hatton, 2014; Mitchell, 2007; Nicolau, 2005; Pérez Moreda et al., 2015). La historiografía ha debatido sobre sus causas. McKeown (1978) argumentó que este descenso estuvo impulsado por mejoras en la dieta alimentaria y el nivel de vida, y solo parcialmente por mejoras en la dotación de infraestructuras de salud pública. Otros autores como Szreter $(1988,2002)$, por el contrario, señalaban que Mckeown había exagerado la importancia de las mejoras nutricionales, infravalorando la contribución de las reformas sanitarias y la prevención de las enfermedades infecciosas. Los estudios realizados muestran que avanzado el siglo xx los adelantos médicos y las políticas de salud pública fueron tan importantes como las mejoras en el consumo alimentario y los avances tecnológicos (Bell y Millward, 1998; Cutler y Miller, 2005).

La evidencia histórica muestra que en el largo plazo el descenso de la mortalidad y la modernización demográfica no fue sostenido y estuvo jalonado por épocas de retroceso y avances. Guerras, malas cosechas y crisis de subsistencias, prevalencia de las enfermedades infecciosas o el embate de las crisis epidémicas fueron las principales causas que alteraron el ritmo de descenso de la mortalidad en muchos países (Floud et al, 2011, 2014; Pérez Moreda et al., 2015; Sanz Gimeno y Ramiro Fariñas, 2002a). La fiebre amarilla, por ejemplo, mató a principios del siglo xix en la ciudad de Cádiz a más de 19000 personas. Esta enfermedad, un mal endémico de las zonas tropicales que se trasmite por picadura de mosquitos, se extendió con virulencia por una ciudad que entonces mantenía una rica prosperidad apoyada por el comercio americano de ultramar. En los distintos brotes la ciudad aplicó el cierre de barrios, el aislamiento de los enfermos menos graves en lazaretos y los más afectados en hospitales (Martín, 2012; Ramírez, 2012). Las décadas posteriores vinieron marcados a nivel nacional y regional por varias fluctuaciones en torno a crisis de subsistencias y epidémicas, destacando entre estas últimas las de cólera de 1834,1855 y 1885, y las de gripe, como la que afectó a la ciudad de Madrid entre 1889 y 1890 (Gozálvez, 2003; Moll et al, 2014; Pérez Moreda et al., 2015; Sanz Gimeno y Ramiro Fariñas, 2002a; García Ferrero, 2018). La más mortífera, sin embargo, tuvo lugar en los albores del siglo xx.

La pandemia de gripe de principios del siglo xx, que se extendió entre 1918 y 1920 y que llegó a medio mundo, infectando a quinientos millones de personas (la tercera parte de la población mundial de la época), mató a cincuenta millones (Ansart et al, 2009; Spinney, 2010; Ferreira, 2020). A pesar de su elevada letalidad, la peor pandemia de los tiempos modernos dejó poca huella en la memoria cultural de los coetáneos, explicable por haber coincidido con el final de la Primera Guerra Mundial y el inicio de los "felices años veinte» (Altares, 2020). En el ámbito económico el impacto fue más perceptible. Según un estudio reciente, la caída en la actividad económica fue persistente en Estados Unidos. La epidemia provocó una caída media del 18\% en la producción industrial estadounidense. Las zonas más expuestas, que sufrieron una mayor mortalidad y volumen de quiebras empresariales y hogares, permanecieron más deprimidas en relación con las áreas menos afectadas hasta comienzos de los años veinte (Correia et al., 2020).

En España, la pandemia mató entre 1918 y 1920 alrededor de 260000 personas, provocando el mayor descenso vegetativo del siglo xx (Echevarri, 1993) ${ }^{1}$. Si bien la mayoría de los fallecidos eran jóvenes sanos de entre 20 y 40 años, y se concentró, además, el 75\% de las muertes en el otoño de 1918, la epidemia elevó también las tasas de mortalidad infantil de aquel año y subsiguientes (Gómez Redondo, 1992; Erkoreka, 2010). Entre 1916 y 1918 la mortalidad infantil pasó de una tasa media de 151,1 a 183,0 por 1000 nacidos vivos. La tasa alcanzada en 19161917 no se recuperó hasta aproximadamente 1921 (Nicolau, 2005, p. 131). El resultado del aumento de la tasa de muertes se reflejó en el estancamiento de la esperanza de vida al nacer. Si entre 1900 y 1910 la esperanza de vida de los españoles aumentó en 7 años (de 33,9 a 40,9 años), entre 1910 y 1920 se estancó (de 40,9 a 40,3 años), ocurriendo lo mismo entre la población masculina (de 42,6 a 42,1 años) y femenina (de 41,7 a 41,2 años) (Nicolau, 2005: 86). En los años veinte, superada la pandemia, la esperanza de vida de la población masculina, femenina y total española volvió a aumentar ( 8,8 años a nivel nacional) (id.). Para contener la expansión del virus, las autoridades españolas ordenaron desinfecciones masivas y medidas de choque que supusieron el cierre de fronteras, universidades y escuelas, el control del tráfico ferroviario o el establecimiento de cordones sanitarios marítimos (Echevarri, 1993).

Aunque desde la década de 1990 existen diversos estudios que han analizado el impacto de la epidemia de gripe de 1918 a nivel nacional y urbano y rural (Echevarri, 1993; Álvarez et al., 2008; Chowell et al., 2014; Cilek et at., 2018; Durán, 2020; Erkoreka, 2006; Martínez y Barona, 1996; Sanz Gimeno y Ramiro Fariñas, 2002a), este artículo profundiza sobre los efectos demográficos y económicos que la pandemia de gripe tuvo en la España rural interior. Para ello, analiza el caso del municipio de Hellín, en la provincia de Albacete (figura 1). Con 18799 habitantes en 1920, Hellín era una agrociudad apoyada en la clásica trilogía mediterránea (cereales, vid y olivos) y la ganadería extensiva, y era el segundo municipio más poblado de la provincia tras la capital, lo que podría considerarse un microcosmos representativo de La Mancha meridional (Cañabate, 2015, 2016). El estudio epidemiológico y su comparación con otros contextos de mayor referencia permitirá saber si el caso de Hellín siguió pautas generales o, por el contrario, mostró aspectos singulares en la evolución de la pandemia, como la mortalidad por causas de muerte, enfermedades infecciosas o estructura de edad.

183121 personas por la epidemia de 1918 frente a los 50266 ocasionados por la Guerra Civil española. 


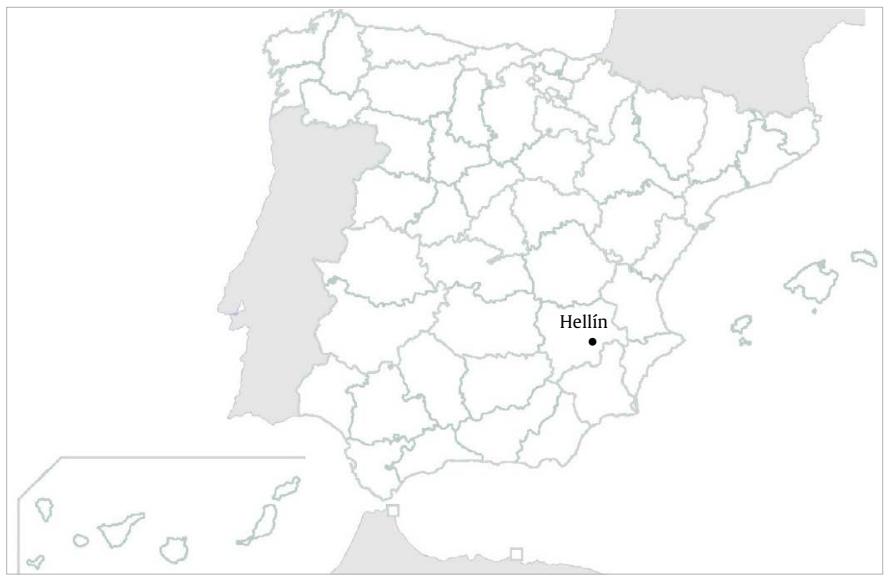

Figura 1. Localización de Hellín

Fuente: elaboración propia.

El artículo se ha estructurado en cuatro apartados: el primero ha presentado el objetivo del trabajo en esta introducción; el segundo describe las fuentes de estudio, los datos de la muestra y la metodología utilizada; el tercero presenta los resultados obtenidos y la discusión, analizando, en primer lugar, el impacto que la epidemia de gripe de 1918 tuvo sobre el proceso de transición demográfica y la morbimortalidad en Hellín, comparando su incidencia con lo acontecido en otros contextos geográficos, y, en segundo lugar, los posibles efectos económicos que pudo tener la pandemia. El trabajo finaliza con el cuarto apartado, dedicado a las conclusiones.

\section{Fuentes, datos y metodología}

Para analizar el impacto demográfico de la pandemia de gripe de 1918 en Hellín hemos utilizado como fuente principal la información que ofrece el Registro Civil de Hellín (RCH). Los libros de nacimientos y defunciones de dicho registro hacen posible trabajar con información abundante y fiable relacionada con los nacimientos, las muertes, el sexo y edad del fallecido y las causas del fallecimiento ${ }^{2}$. Además, las expresiones que informan sobre dicha causa de muerte resultan plenamente fiables, pues provienen de las certificaciones expedidas por el médico que registraba la defunción de cada individuo. También se han consultado los datos de población absoluta recogidos en los censos de población.

Para estudiar el posible impacto que la pandemia pudo tener en la economía hellinera, el artículo ha utilizado tres tipos de fuentes. Por un lado, la fuente primaria de las matrículas de la contribución industrial y de comercio ${ }^{3}$, que aporta información sobre el número de matriculaciones en el ámbito industrial y comercial; por otro lado, y para evaluar el posible impacto de la epidemia en el sector agrario, se han utilizado los datos de producción de cereales, vino y aceite en la provincia

\footnotetext{
2 También aparece información sobre la profesión de los varones fallecidos, pero los datos son tan escasos que nos impide analizar el gradiente social de la mortalidad por gripe en Hellín.

${ }^{3}$ Para más información sobre esta fuente primaria ver Nadal (1987) y Beltrán (1999).
}

de Albacete elaborados por el Grupo de Estudios de Historia Rural (GEHR, 1991). Por último, para estimar su impacto sobre el nivel de vida de la clase trabajadora se han empleado los datos de los salarios nominales abonados a los obreros agrícolas eventuales en el área vitivinícola del sureste español, asî como el índice de precios al por menor de los artículos de primera necesidad en los pueblos de la región de Murcia (Martínez Soto, 2002, 2006).

Finalmente, se han consultado los libros de actas del Pleno del Ayuntamiento de Hellín. Esta fuente primaria, de carácter político-administrativo, es de sumo interés porque ofrece información directa sobre la incidencia que tuvo la pandemia de gripe en el municipio, y también permite estudiar qué políticas de salud pública se adoptaron para hacer frente a la epidemia gripal, así como la incidencia que tuvieron en las condiciones de vida y salud de la población.

Los datos de defunciones y causas de muerte en el municipio de Hellín son de gran calidad. La muestra asciende a un total de 13961 decesos, de los que el 100\% contiene información sobre la causa de muerte (tabla 1), sumando un total de noventa expresiones diagnósticas diferentes durante el periodo de estudio.

Para analizar las expresiones diagnósticas relacionadas con la gripe que provocó la pandemia y las posibles enfermedades que se vieron afectadas por el impacto de la misma, es necesario, a nivel metodológico, utilizar una clasificación de enfermedades que nos permita la homologación entre las rúbricas existentes. Con ello se busca facilitar el análisis evolutivo de las enfermedades que nos interesan analizar y la comparación de resultados de mortalidad provocada por las mismas. En este sentido, se ha buscado la correspondencia entre las clasificaciones de enfermedades elaboradas por Jacques Bertillon y Thomas McKeown. El primero utiliza un criterio anatómico para las enfermedades (Bertillon, 1899), mientras que el segundo emplea criterios etiológicos, distinguiendo entre enfermedades infecciosas y no infeccionas (McKeown, 1978).

Aunque el objetivo principal es estudiar el impacto de la gripe de 1918, al ser esta pandemia una enfermedad epidémica de transmisión aérea, hemos tenido en cuenta también las principales enfermedades respiratorias. Estas causas de muerte no se asumieron como fallecimientos provocados directamente por la gripe, aunque en muchos casos pudieron corresponder a complicaciones derivadas de la misma. Igualmente, se ha decidido incluir el análisis de las diarreas y gastroenteritis, pues aunque su transmisión no fuera aérea sí es infecciosa, y en muchos casos los diagnósticos estaban relacionados con la infección gripal (tabla 2). Además, en esta época eran la principal causa de muerte entre los menores de cinco años y continuó siéndolo después de la pandemia (Gómez Redondo, 1992; Sanz Gimeno y Ramiro Fariñas, 2002b; Pérez Moreda et al., 2015).

A nivel metodológico también se ha procedido a solucionar dos tipos de problemas adicionales. El primero atiende a la denominada "moda diagnóstica", por la cual algunas rúbricas adquieren cierto peso en momentos determinados, atrayendo bajo su denominación a otras enfermedades similares. El segundo versa sobre la confusión provocada por rúbricas que combinan síntoma y localización anatómica, decidiendo asignar dicha rúbrica al apartado de enfermedades no infecciosas propia de esa zona anatómica (Bernabeu Mestre et al., 2003). 
Tabla 1

Número de defunciones en el municipio de Hellín según tipo de enfermedad, 1900-1929

\begin{tabular}{|c|c|c|c|c|c|c|c|}
\hline \multirow[b]{3}{*}{ TRIENIO } & \multicolumn{4}{|c|}{ ENFERMEDAD } & & & \multirow[b]{3}{*}{ TOTAL } \\
\hline & \multicolumn{2}{|c|}{ INFECCIOSA } & \multicolumn{2}{|c|}{ NO INFECCIOSA } & \multicolumn{2}{|c|}{ VEJEZ } & \\
\hline & H & $\mathbf{M}$ & H & M & $\mathbf{H}$ & $\mathbf{M}$ & \\
\hline $1900-1902$ & 479 & 455 & 158 & 173 & 5 & 14 & 1.284 \\
\hline 1903-1905 & 473 & 405 & 208 & 212 & 12 & 26 & 1.336 \\
\hline 1906-1908 & 496 & 430 & 207 & 193 & 22 & 23 & 1.371 \\
\hline 1909-1911 & 565 & 470 & 191 & 214 & 18 & 21 & 1.479 \\
\hline $1912-1914$ & 480 & 460 & 188 & 189 & 20 & 24 & 1.361 \\
\hline 1915-1917 & 498 & 428 & 165 & 170 & 23 & 13 & 1.297 \\
\hline 1918-1920 & 747 & 649 & 239 & 213 & 25 & 41 & 1.914 \\
\hline $1921-1923$ & 462 & 416 & 216 & 204 & 22 & 28 & 1.348 \\
\hline 1924-1926 & 406 & 379 & 182 & 206 & 28 & 42 & 1.243 \\
\hline 1927-1929 & 435 & 451 & 191 & 174 & 37 & 40 & 1.328 \\
\hline
\end{tabular}

Fuente: libros de defunciones del RCH. Elaboración propia.

Tabla 2

Relación y correspondencia de las enfermedades analizadas

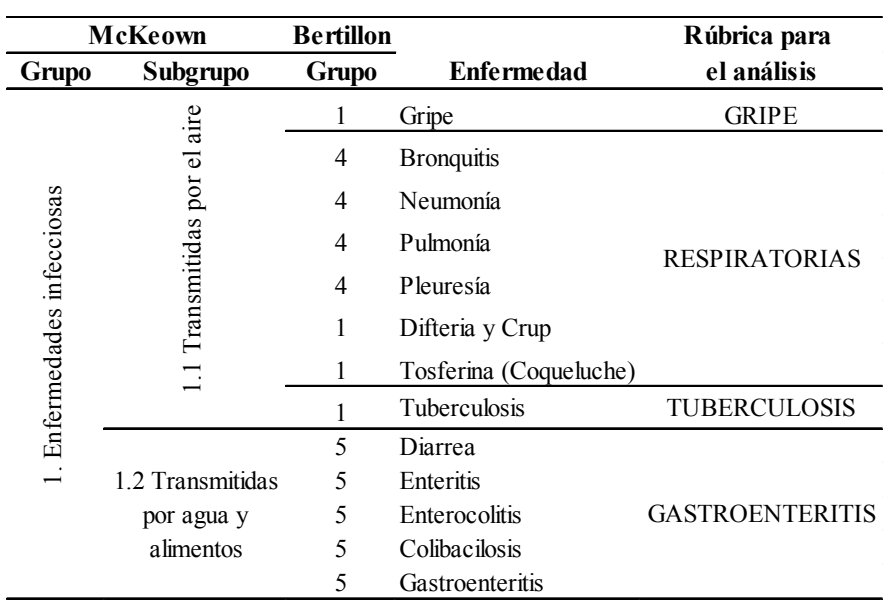

Fuente: Bertillon (1899) y McKeown (1978). Elaboración propia.

Por último, y para completar el análisis demográfico, hemos empleado el denominado índice de Dupâquier, que tiene como objetivo comprobar la existencia o no de una crisis de mortalidad asociada a la pandemia de gripe de 1918 (Álvarez et al., 2008). Partiendo de la premisa de que una crisis de mortalidad se puede definir como un episodio de mortalidad excepcionalmente alto respecto a su entorno espacio-temporal, este método nos ofrece una expresión matemática $(I=(D-M) s-1)$ que determina la intensidad de la mortalidad durante un intervalo definido, donde $I$ es la intensidad de la crisis, $D$ es el número de muertos en el año estudiado, y $M$ y $s$ son la media y la desviación típica de las defunciones de todos los años sin incluir el año estudiado, el anterior y el posterior. El índice de Dupâquier toma siempre valores positivos para años con mortalidades superiores a la media y, según su magnitud, permite clasificar la crisis de mortalidad de la siguiente manera:

\begin{tabular}{|c|c|c|c|c|c|c|}
\hline & \multicolumn{4}{|c|}{ Crisis } & \multirow{2}{*}{$\begin{array}{l}\text { Gran } \\
\text { Crisis }\end{array}$} & \multirow{2}{*}{$\begin{array}{c}\text { Crisis } \\
\text { Catastrófica }\end{array}$} \\
\hline & Menor & Media & Fuerte & Importante & & \\
\hline $\begin{array}{l}\text { Índice } \\
\text { Dupâquier }\end{array}$ & $1-2$ & $3-4$ & $5-8$ & $9-16$ & $17-32$ & $>32$ \\
\hline
\end{tabular}

En este trabajo hemos aplicado el índice de Dupâquier a la mortalidad del año 1918 para el periodo de referencia del primer tercio del siglo $\mathrm{xx}$, periodo para el cual disponemos de una serie homogénea y completa para esa variable demográfica.

\section{Resultados y discusión}

\subsection{Los comienzos de la transición demográfica en Hellín}

Durante el primer tercio del siglo xx, el municipio de Hellín, como ocurría en el resto de Castilla-La Mancha y España, estaba inmerso en un proceso de transformación demográfica caracterizado por un lento declive de la mortalidad y las enfermedades infecciosas (Camacho, 1999). La figura 2 muestra la evolución de la población y de las tasas brutas de natalidad y mortalidad en Hellín durante el primer tercio del siglo xx. Se observa que la población hellinera creció en casi diez mil habitantes, si tomamos como referencia los datos censales de 1900 y 1930. El crecimiento demográfico fue más evidente en 
la primera y tercera década, explicable por el mayor diferencial en la caída de las tasas brutas, y menor en la segunda década, achacable al impacto vegetativo ocasionado por la epidemia de gripe de 1918.

Las tasas anuales de nacimientos y muertes de la población ilunense siguieron, desde comienzos de siglo hasta 1930, una tendencia de descenso (figura 2). En ese tiempo, las tasas brutas de natalidad y mortalidad disminuyeron cerca de un $24,5 \%$ y $38,8 \%$ respectivamente, resultado de la mejora relativa de la alimentación y de las infraestructuras higiénico-sanitarias (Cañabate, 2015, 2016). Así, se pasó de una tasa del 47,1 al 35,5 por 1000 habitantes en el caso de los alumbramientos, y del 30,1 al 18,4 por 1000 habitantes en las defunciones.

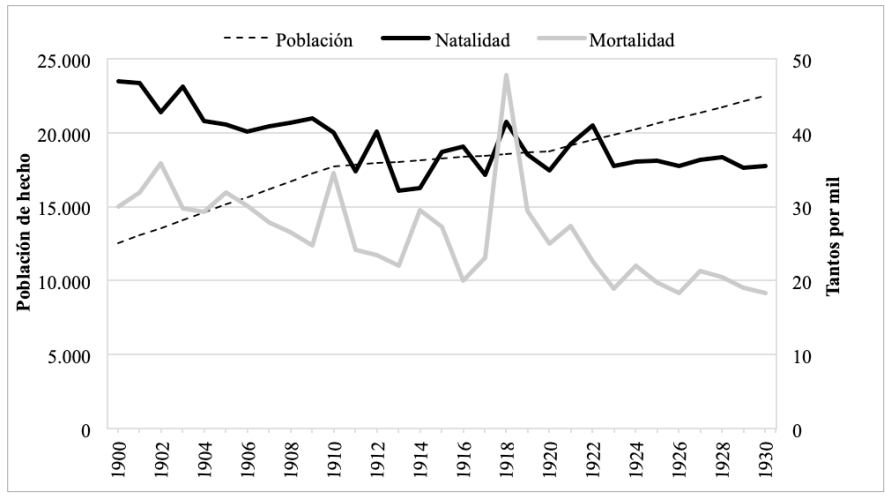

Figura 2. Evolución de las tasas brutas de natalidad y mortalidad en Hellín, 1900-1930 (por 1000 habitantes).

Nota: La población anual se ha estimado a partir de una interpolación lineal de los censos de población de 1900, 1910, 1920 y 1930. Se trata, como es lógico, de un valor aproximado.

Fuente: censos de población del INE y libros de defunciones del RCH. Elaboración propia.

El descenso de la mortalidad, sin embargo, y como ocurrió en otras poblaciones españolas, no fue sostenido, viéndose interrumpido por la pandemia de gripe de 1918, que elevó las tasas de muertes de aquel año del 23,2 \% en 1917 al 47,9\% en 1918, mientras que en Madrid la tasa bruta de mortalidad en 1918 fue del 29,4 \% (Erkoreka, 2010). Como consecuencia de ello, el resultado obtenido de aplicar el índice de Dupâquier, con un valor de 8`3, sugiere que el municipio de Hellín sufrió una crisis fuerte de mortalidad asociada a la epidemia de gripe. Superada la epidemia, la tasa de mortalidad retomó su tendencia descendente, alcanzando su cifra más baja a finales de los años veinte (figura 2 ).

El avance del proceso de modernización demográfica en España, al igual que aconteciera con otros países de su entorno geográfico próximo, se debió principalmente a la disminución de la mortalidad en la infancia (Gómez Redondo, 1992; Nicolau, 2005; Pérez Moreda et al., 2015). Hellín no fue una excepción. Como muestra la figura 3, la mortalidad infantil en esta población de La Mancha meridional conoció un notable descenso, pasando de una tasa de 196,3 por 1000 nacidos vivos en 1900 a situarse en el 131,1\% en 1930. Sin embargo, y como ocurrió con la mortalidad general, este descenso fue irregular, siendo interrumpido en dos ocasiones. Por un lado, por las crisis epidémicas de 1910 (sarampión) y 1914-1915 (gastroenteritis), incrementando esta última las tasas de mortalidad hasta el 269,7 por 1000 nacidos vivos y, por otro, por la epidemia de gripe de 1918, que disparó la tasa de mortalidad infantil de 1918 a 1919 hasta casi los 300 por 1000 nacidos vivos. Aunque la pandemia de gripe afectó más a los jóvenes sanos de 20 a 40 años, la figura 3 revela que la población infantil no fue inmune a la enfermedad epidémica.

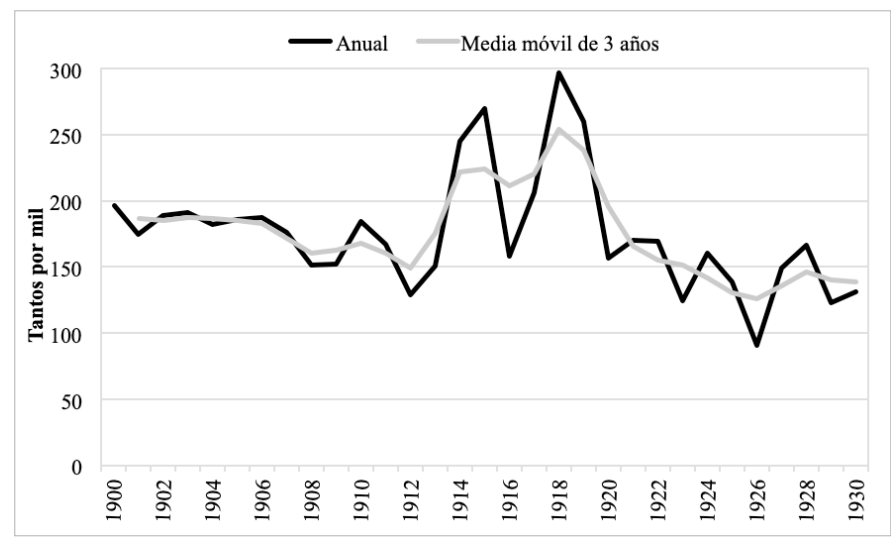

Figura 3. Evolución de la tasa de mortalidad infantil en Hellín, 1900-1930 (por 1000 nacidos vivos)

Fuente: libros de defunciones del RCH. Elaboración propia.

A pesar de ello, y como han apuntado diversos autores, la correlación de la gripe con muchos óbitos infantiles fue indirecta. Ello es debido a que gran parte de los fallecimientos no obedecieron solo a la gripe, sino también a otras enfermedades de carácter respiratorio (como bronquitis o bronconeumonía) o digestivo (como diarreas y enteritis) que afectaron especialmente a los niños, ya debilitados antes por los efectos de la pandemia tanto sobre sus madres ${ }^{4}$ como sobre ellos mismos (Echeverri, 1993, p. 120; Sanz Gimeno y Ramiro Fariñas, 2002a, p. 378). Sea lo que fuere, es evidente que el aumento de la mortalidad infantil durante la influenza mermó la progresión de la esperanza de vida al nacer de la población española, que descendió ligeramente en la década de 1910 , haciendo incluso que el índice de desarrollo humano (IDH) aumentara muy poco entonces (Escudero y Simón, 2003, pp. $538,541-542$ ). En nuestro caso, como puede verse en la figura 4, la epidemia de gripe de 1918 tuvo también un efecto negativo sobre la edad media de supervivencia de la población masculina, femenina y total hellinera, utilizada en este artículo como un proxy de la esperanza de vida. Entre 19161917 y 1918, la edad media de supervivencia de los hombres y mujeres de Hellín pasó de los 44,5 y 43,8 años a los 29,8 y 31,8 años, respectivamente. Y para el conjunto de la población la regresión fue igualmente severa, cayendo entre 1916-1917 y 1918 de 44,2 años a 30,8 años. Al término de la pandemia, y durante los años veinte, la tasa de supervivencia mejoró significativamente.

\footnotetext{
${ }^{4}$ Almond (2006), a partir de datos censales de Estados Unidos de 196080, reveló que las cohortes en el útero durante la pandemia de 1918 mostraron un logro educativo reducido, mayores tasas de discapacidad física y menor nivel socioeconómico en comparación con otras cohortes
} de nacimiento. 


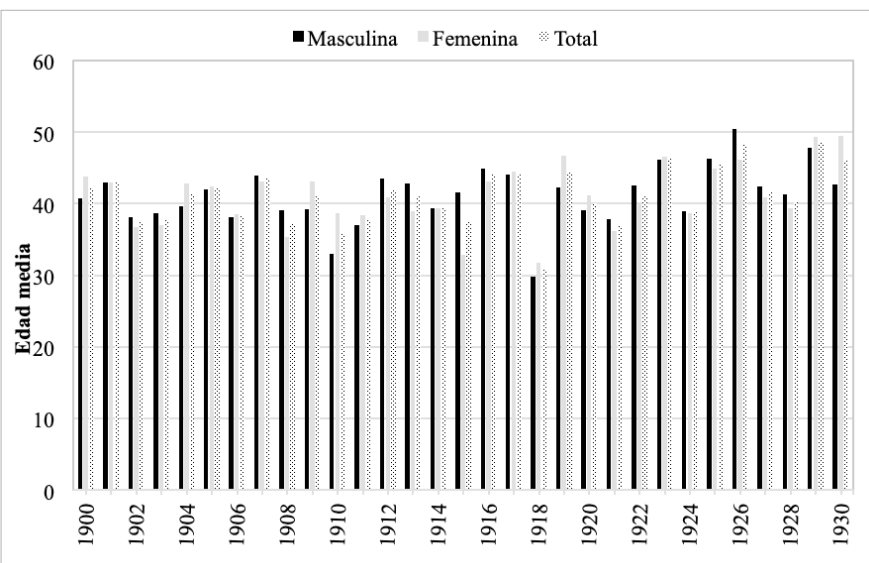

Figura 4. Edad media de supervivencia en Hellín, 1900-1930.

Nota: Este indicador se ha obtenido calculando la edad promedio de fallecimientos anuales a partir de las edades de los decesos contenidas en los Libros de Defunciones. Los resultados logrados se aproximan a los cálculos de esperanza de vida obtenidos en otros trabajos (Blanes, 2007).

Fuente: libros de defunciones del RCH. Elaboración propia.

\subsection{El impacto de la epidemia de gripe de 1918 en la morbimor- talidad}

Durante el primer tercio del siglo xx el municipio de Hellín se encontraba inmerso en un proceso de transición epidemiológica, entre la erradicación de las tradicionales crisis epidémicas, como el cólera y la viruela ${ }^{5}$, y la reducción de las enfermedades infecciosas. En este contexto, las causas de muerte continuaron siendo diversas y mantenían una estrecha relación con la falta de higiene, pobreza y desnutrición. Según Echevarri (1993, p. 103), las poblaciones empobrecidas, desnutridas, en malas condiciones higiénico-sanitarias y con poco acceso a la asistencia médica, padecen más infecciones.

La elevada mortalidad ordinaria que presentaba Hellín a principios del siglo xx estuvo vinculada a la gran incidencia de las enfermedades infectocontagiosas y las crisis epidémicas. Al comienzo de siglo, las enfermedades infecciosas suponían el $72,7 \%$ de la mortalidad del municipio. Esta incidencia paulatinamente fue decreciendo hasta que la pandemia de gripe de 1918 elevó nuevamente la mortalidad infecciosa al 79,7\%. Desde entonces, y hasta 1929, descendieron al 65,4\%.

Tras las dos últimas epidemias de cólera de 1885 y 1890, otras enfermedades epidémicas protagonizaron la mortalidad catastrófica en las dos primeras décadas del siglo xx. Destacan los episodios de mortalidad de 1910 -vinculado con el sarampión y enfermedades gastroentéricas- y 1914 -relacionado con la bronquitis, viruela y meningitis- (Cañabate, 2015). Con todo, la última gran oscilación que aparece es la correspondiente a la epidemia gripal de 1918, que provocó una elevación repentina de la tasa de muertes. En ese año la mortalidad general aumentó en Hellín un 99,3\% con respecto al periodo de 1913-1917, mientras que en ciudades próximas como Albacete (91,7\%), Murcia (90,4\%) y en el conjunto de

\footnotetext{
${ }^{5}$ Durante un pleno celebrado en mayo de 1914, se instó al alcalde para que "adoptara las medidas sobre higiene y salubridad requeridas para evitar el contagio de viruela por el municipio". Archivo Municipal de Hellín (AMH), Gobierno, A-59.
}

España $(95,1 \%)$ se produjo un incremento porcentual relativamente menor (Chowell et al., 2014).

Si comparamos la mortalidad de 1919-1930 con la de 1918 , se observa una caída del $54,2 \%$, recuperándose la tendencia descendente que había predominado hasta 1917 (figura 2). Este comportamiento indica que el impacto de la epidemia de 1918 fue transitorio y no provocó un cambio en los patrones de mortalidad del municipio, aunque si ralentizó el proceso de transición demográfica y epidemiológica.

Entre 1900 y 1917, el $2 \%$ de los fallecimientos acaecidos en Hellín fueron a causa de la gripe, mientras que en 1918 las defunciones por esta enfermedad epidémica ascendieron al $12 \%$, lo que se traduce en una tasa de mortalidad por gripe del 5,75 \% para ese año. El exceso de mortalidad provocado por la epidemia gripal respecto al periodo previo de 1913-17, fue del 5,39\%, una tasa ligeramente superior al exceso registrado en la ciudad de Madrid, que fue del 5,27 \% (Erkoreka, 2010). A partir de 1919, y hasta 1929, el promedio de muertes por gripe desciende al 1,3\%, con tasas de mortalidad prepandémicas.

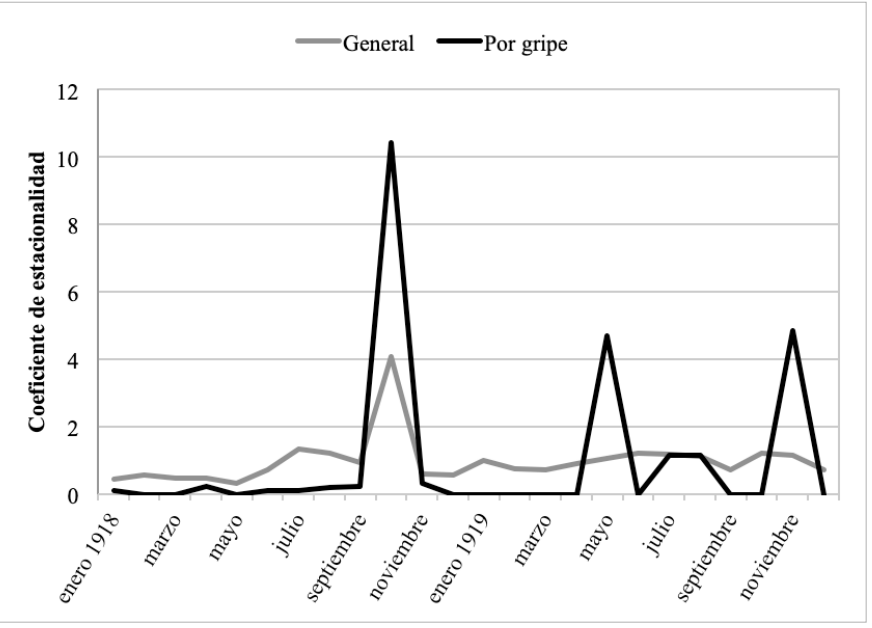

Figura 5. Coeficientes de estacionalidad de la mortalidad general y por gripe en Hellín, enero de 1918-diciembre de 1919.

Fuente: libros de defunciones del RCH. Elaboración propia.

La figura 5 muestra los coeficientes de estacionalidad de la mortalidad general y por gripe en Hellín entre enero de 1918 y diciembre de 1919. La comparación es interesante porque permite observar la estacionalidad e incidencia que tuvieron las olas pandémicas en el municipio. Mientras en la ciudad de Madrid la gripe y las enfermedades respiratorias asociadas a ella provocaron durante la primera oleada (mayo-junio de 1918) una tasa de mortalidad de $1,31 \%$ (Erkoreka, 2010), esta tuvo una baja incidencia en Hellín (0,86\%). En este contexto, el Ministerio de Gobernación dirigió una circular a los gobernadores provinciales aconsejando que se evitaran grandes aglomeraciones públicas en los pueblos con motivo de ferias y fiestas, sugiriendo su aplazamiento ${ }^{6}$. Esta recomendación, sin embargo, no se cumplió en Hellín a raíz de las aglomeraciones que se formaron a causa del entierro

\footnotetext{
${ }^{6}$ ABC de 23 de septiembre de 1918. Como motivo de esta circular, Hellín suspendió la feria a finales de septiembre y comienzos de octubre. Libro de actas del Ayuntamiento de Hellín, 1918-1919. AMH, Gobierno, A_00171_002.
} 
de Tesifonte Gallego ${ }^{7}$, cuyo funeral a finales de septiembre de 1918 reunió a multitud de vecinos. Este hecho, muy probablemente, aceleró los contagios de gripe entre la población congregada. La Junta Municipal de Sanidad (JMS), ante el aumento de decesos por gripe constatados a principios de octubre, acordó «las medidas sanitarias necesarias para atenuar la epidemia de gripe», destacando el aislamiento de enfermos, la desinfección y ventilación de las viviendas y la mejora de las condiciones higiénico-sanitarias de lavaderos públicos y la conducción de agua potable ${ }^{8}$.

El aumento de la mortalidad general en octubre de 1918 coincidió con la segunda oleada de la epidemia, que como muestra la figura 5 tuvo una enorme incidencia en Hellín. La elevada morbimortalidad, causada principalmente por la enfermedad epidémica, fue también el resultado de la incidencia de las enfermedades respiratorias y gastroentéricas influenciadas por ella ${ }^{9}$. En perspectiva comparativa, si durante los meses de octubre y noviembre de 1918 las muertes provocadas por la gripe y las afecciones respiratorias representaron en la ciudad de Madrid una tasa de mortalidad del 0,62 y del 1,79\%, respectivamente (Erkoreka, 2010), en Hellín las tasas de mortalidad por dichas causas se elevaron hasta los 5,27 y 5,53\%. Dos posibles factores podrían explicar estas diferencias territoriales. La primera es que gran parte de la población anciana de Madrid ya había estado expuesta a otras pandemias recientes, como la epidemia de gripe de 1889-90 (García Ferrero, 2018), mientras que la segunda apuntaría a la mayor incidencia que tuvo en la capital la primera oleada de gripe, en la primavera de 1918. Ambos factores pudieron proveer de una mayor inmunidad viral en la ciudad, provocando de este modo que la segunda oleada pandémica tuviera una menor incidencia en la morbimortalidad (Cilek et al., 2018).

Tan grave fue la situación en el mes de octubre, que el día 1 de noviembre de 1918 el libro de actas de Pleno del Ayuntamiento de Hellín recogió el acuerdo de instruir expediente administrativo al médico de una pedanía del municipio "por haberse ausentado durante la epidemia de gripe dejando en completo abandono a los muchos enfermos que tiene obligación ineludible de asistir, dándose hasta el caso de que algún cadáver haya permanecido insepulto varios días por falta de la expedición del certificado médico correspondiente». La figura 5 también refleja el impacto que la gripe tuvo en Hellín durante los meses de mayo y noviembre de 1919, aunque su incidencia sobre la mortalidad general fue menos relevante respecto a lo acontecido en el mes de octubre de 1918.

Como se indicó en el apartado de fuentes, se han analizado cuatro grupos de enfermedades: la gripe, las afecciones respiratorias, la tuberculosis y las gastroenteritis, por ser las enfermedades infecciosas que mayor mortalidad causaban hasta ese momento a nivel nacional, especialmente entre la población infantil (Castelló, 2011; Sanz Gimeno y Ramiro Fariñas, 2002b). La figura 6 muestra cómo los efectos de la epidemia de gripe se extendieron a otras enfermedades infecciosas de transmisión aérea, por agua y alimentos.

\footnotetext{
7 Personalidad ilustre, fue periodista, abogado y diputado a Cortes por Hellín en 1901, llegando a ser director general de Agricultura y principal promotor para que Hellín consiguiera el título de ciudad en 1898.

${ }^{8}$ Libro de actas de la JMS, 6 de octubre de 1918. AMH, A_00095_003.

${ }_{9}$ Aunque con desigual intensidad, la mayor incidencia de la segunda ola pandémica también se ha documentado en otras zonas del país como Asturias (Álvarez, et al., 2008), Badajoz (Durán, 2020), Madrid (Erkoreka, 2010; Chowell et al., 2014; Cilek et al., 2018) o el País Vasco (Erkoreka, 2006).
}

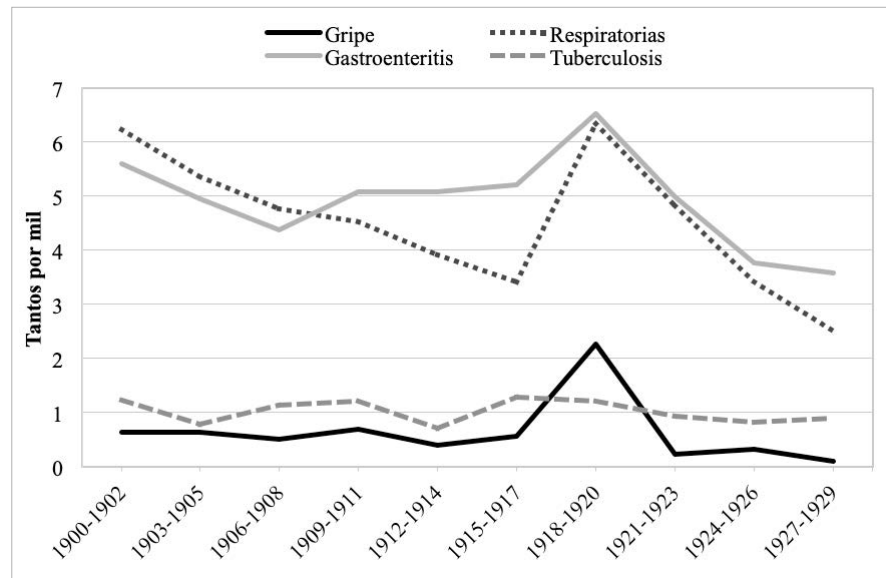

Figura 6. Tasas brutas de mortalidad por causas de defunción en Hellín, 1900-1929 (por 1000 habitantes).

Nota: La población anual se ha estimado a partir de una interpolación lineal de los censos de población de 1900, 1910, 1920 y 1930. Se trata, como es lógico, de un valor aproximado.

Fuente: libros de defunciones del RCH y censos de población del INE. Elaboración propia.

Si analizamos la evolución de estos cuatro grupos de enfermedades, en comparación con el período prepandémico se observa un aumento de la mortalidad durante el trienio de 1918-20, y un posterior descenso a partir de 1921-23 (figura 6). Este hecho vendría a confirmar que Hellín estaba inmerso en el proceso de transición epidemiológica con la reducción de las enfermedades infecciosas. Se evidencia claramente cómo en plena pandemia la mortalidad por causa de gripe, enfermedades respiratorias y grastroentéricas se incrementó, y cómo a partir del trienio 1921-23 dicha mortalidad experimentó una gran caída. Durante este periodo, por el contrario, la tuberculosis apenas sufrió modificación alguna, evidenciando en todo caso un leve descenso desde el trienio 1915-17.

Por causa de muerte, entre 1900 y 1917 la gripe mantuvo unas tasas de mortalidad de 0,58 por 1000 habitantes. Durante el período pandémico de 1918-20 las personas fallecidas por esta causa aumentaron hasta los 128 , de los cuales 107 lo hicieron en 1918 y 95 en octubre, lo que supuso que la tasa de mortalidad por esta enfermedad ascendiera hasta el 2,28 por cada 1000 habitantes. Su incidencia, como se puede ver en la figura 6, se extendió también a las enfermedades infecciosas de transmisión aérea que conforman el grupo llamado respiratorias. Hasta 1917 se observa un claro descenso de las mismas, interrumpido bruscamente en 1918-20 al alcanzar una tasa de mortalidad de 6,35 por 1000 habitantes. Las tasas de 1915-17 no se volvieron a recuperar hasta 1924-26, y en su descenso resultó fundamental la prevención del contagio y la inmunización, así como las mejoras relativas a las condiciones de la vivienda, su ventilación y la separación de los animales y los miembros de la familia (Pérez Moreda et al., 2015).

En el caso de las enfermedades infecciosas transmitidas por agua y alimentos, reunidas bajo el epígrafe de gastroenteritis, la figura 6 revela que el periodo de mayor mortalidad de este grupo de afecciones fue el comprendido entre 1909-11 y 192123. El dato, como ha apuntado la literatura especializada, estaría relacionado con una dieta alimentaria insuficiente y unas penosas condiciones higiénico-sanitarias (Pérez Moreda et al., 2015; Sanz Gimeno y Ramiro Fariñas, 2002a, 2002b). En Hellín, 
Tabla 3

Tasas brutas de mortalidad según causa, sexo y edad de muerte en Hellín, 1918 (por 1000 habitantes)

\begin{tabular}{|c|c|c|c|c|c|c|}
\hline & \multicolumn{2}{c}{ GRIPE } & \multicolumn{2}{c|}{ RESPIRATORIAS } & \multicolumn{2}{c|}{ GASTROENTERITIS } \\
\hline & Hombres & Mujeres & Hombres & Mujeres & Hombres & Mujeres \\
\hline $\mathbf{1}$ & 0,05 & 0,05 & 1,29 & 0,48 & 1,24 & 0,86 \\
\hline $\mathbf{1}$ a $\mathbf{4}$ & 0,16 & 0,32 & 1,94 & 1,45 & 2,90 & 3,07 \\
\hline $\mathbf{1 5}$ a $\mathbf{~ 3 9}$ & 0,11 & 0,38 & 0,27 & 0,38 & 0,11 & 0,59 \\
\hline $\mathbf{4 0}$ a $\mathbf{6 4}$ & 1,61 & 1,77 & 1,88 & 1,72 & 0,11 & 0,05 \\
\hline$\geq \mathbf{6 5}$ & 0,27 & 0,59 & 0,59 & 0,65 & 0,27 & 0,16 \\
\hline Total & 0,32 & 0,11 & 0,38 & 0,43 & 0,16 & 0,22 \\
\hline
\end{tabular}

Fuente: libros de defunciones del RCH. Elaboración propia.

la reducción de las enfermedades gastroentéricas comenzó a hacerse evidente a partir de la segunda mitad de la década de 1920 , gracias a la construcción y mejora de algunas infraestructuras urbanas básicas como el abastecimiento y control de la potabilidad del agua y la red de alcantarillado ${ }^{10}$, además de progresos en la higiene de los alimentos.

$\mathrm{Al}$ igual que no todas las enfermedades respiratorias y gastroentéricas se incrementaron con la misma intensidad, la epidemia de gripe tampoco afectó a la población de la misma manera según edad y sexo. La tabla 3 presentan las tasas brutas de mortalidad por causas, sexo y edad en Hellín en 1918. Se observa que la enfermedad epidémica afectó de forma más grave al grupo de población adulta comprendido entre los 15 y 39 años. De acuerdo con nuestros datos, este colectivo de población representaba el $58,8 \%$ de los fallecidos respecto al total de muertos causados por la gripe. Este rasgo concuerda con los datos mostrados en otros estudios, según los cuales la epidemia de gripe afectó más intensamente a los jóvenes adultos entre los 20 y 39 años (Cilek et al., 2018; Chowell et al., 2014; Durán, 2020). Dentro de este grupo, el más afectado, los datos de Hellín muestran que la tasa de mortalidad fue ligeramente mayor entre las mujeres (1,61 por 1000 habitantes en el caso de los hombres y $1,77 \%$ en el caso de las mujeres) (tabla 3). Los grupos menos afectados fueron los menores de un año, tanto en niños como niñas, los chicos de 5 a 14 años y las mujeres mayores de 65 años, explicable porque los primeros se veían más afectados por otras enfermedades infecciosas, y los segundos porque no constituían un grupo de edad muy numeroso debido a la baja esperanza de vida (Nicolau, 2005; Sanz Gimeno y Ramiro Fariñas, 2002b).

Los niños menores de cuatro años tampoco se vieron muy afectados por la gripe, si bien el porcentaje de enfermedades respiratorias significó el 50,8\% del total de fallecidos en los niños y el 37,9\% en las niñas. Estas enfermedades respiratorias tuvieron también un gran impacto en el grupo de edad comprendido entre los 15 y 39 años, con un número de fallecidos muy similar

\footnotetext{
${ }^{10}$ La canalización de agua potable y el desarrollo del alcantarillado no se llevó a cabo en el municipio de Hellín hasta 1927. Libro de actas del Ayuntamiento de Hellín, AMH, Gobierno, A-0461.
}

entre hombres y mujeres, aunque se documenta una sobremortalidad masculina al tener en cuenta todos los grupos de edad.

La incidencia de las enfermedades reunidas bajo el epígrafe gastroenteritis, afecciones que tradicionalmente tenían mayor impacto entre los menores de 4 años (Gómez Redondo, 1992; Sanz Gimeno y Ramiro Fariñas, 2002b), alcanzaron tasas de mortalidad para estos grupos de edad de $2,9 \%$ entre los niños y 3,07 \% entre las niñas, acumulándose la mayoría de los fallecidos entre los meses de julio y octubre. Tanto en las enfermedades respiratorias como en la gastroenteritis, se advierte una sobremortalidad masculina cuando nos referimos a estos grupos de edad, que en el caso de la gastroenteritis se convierte en femenina cuando se tiene en cuenta todas las edades. La elevada morbimortalidad entre los menores de cuatro años venía determinada por la precariedad de los cuidados maternos e higiénico-sanitarios, así como por la falta de higienización de los alimentos. La lactancia materna ejerció un papel decisivo en la prevención de estas enfermedades infecciosas, pero la aceleración del destete antes de tiempo, la dentición y el paso a una dieta sólida sin la información médica necesaria en lo que respecta a la preparación y mantenimiento de los alimentos, fueron factores que, sin duda, aumentaron el impacto de estas enfermedades infecciosas.

\section{3. ¿Tuvo efectos económicos la pandemia de gripe de 1918 ?}

En esta sección se analiza qué efectos tuvo la pandemia de gripe de 1918 sobre la economía, el bienestar y el nivel de vida de la población hellinera. Para ello, y aunque la evidencia crematística disponible a nivel local es escasa, hemos utilizado tres indicadores económicos, siendo los dos últimos aproximativos de lo que pudo ocurrir en nuestro caso de estudio. En primer lugar, se ha empleado la información primaria de las matrículas de la contribución industrial y de comercio de Hellín, una fuente relevante para analizar los ciclos de actividad económica general, y en particular del sector industrial. En segundo lugar, y para el sector de la agricultura, se han utilizado los datos de producción de cereales, vino y aceite en la provincia de Albacete elaborados por el Grupo de Estudios de Historia Rural (GEHR, 1991). Y en tercer lugar, para evaluar el impacto de la pandemia sobre el nivel de vida de la clase tra- 
bajadora se han empleado los datos de los salarios nominales en el área vitivinícola del sureste español y el índice de precios al por menor de los artículos de primera necesidad en los pueblos de la región de Murcia (Martínez Soto, 2002, 2006).

La figura 7 muestra la evolución que siguieron las matrículas de la contribución industrial y de comercio en Hellín entre 1914 y 1925-26. Se aprecia que los años de la Gran Guerra, que favorecieron a nivel nacional la proliferación de sociedades de nueva creación, la sustitución de importaciones y el impulso de las exportaciones (Carreras y Tafunell, 2010), fomentaron moderadamente la creación de negocios industriales y comerciales en Hellín. En concreto, se pasó de 228 matriculaciones en 1914 a 286 en 1917, lo que supone un aumento del 25,4\%11. Esta tendencia ascendente, sin embargo, se truncó en 1918, al caer levemente las matrículas hasta las 244 , lo que representa un descenso del 14,7\% respecto al año anterior. Esta caída relativa sugeriría un deterioro relativo en los niveles de industrialización y comercialización de la economía hellinera que podría asociarse, en parte, al final del ciclo económico de la guerra mundial, pero también a un posible impacto de la pandemia que, como analizamos en la sección anterior, causó más muertes entre las edades más productivas (jóvenes adultos entre 20 y 40 años). En todo caso, el impacto epidémico debió ser leve, pues los datos muestran que en los tres años siguientes el número de matrículas de la contribución industrial y de comercio aumentaron paulatinamente en Hellín, hasta los 282 en 1920-21, y alcanzando prácticamente el máximo logrado en 1917, con 286 matriculaciones (figura 7). La tendencia se mantuvo estable hasta el bienio 1923-24, con un total de 292 inscripciones, para aumentar de forma exponencial en el trienio 1924-26, donde se alcanzó un máximo de 657 matriculaciones. Entre ambas fechas, el número de matrículas industriales y de comercio creció un $125 \%$, lo que sin duda hay que ponerlo en relación con el ciclo inversor privado que tuvo lugar durante los años de la dictadura de Primo de Rivera (Carreras y Tafunell, 2010).

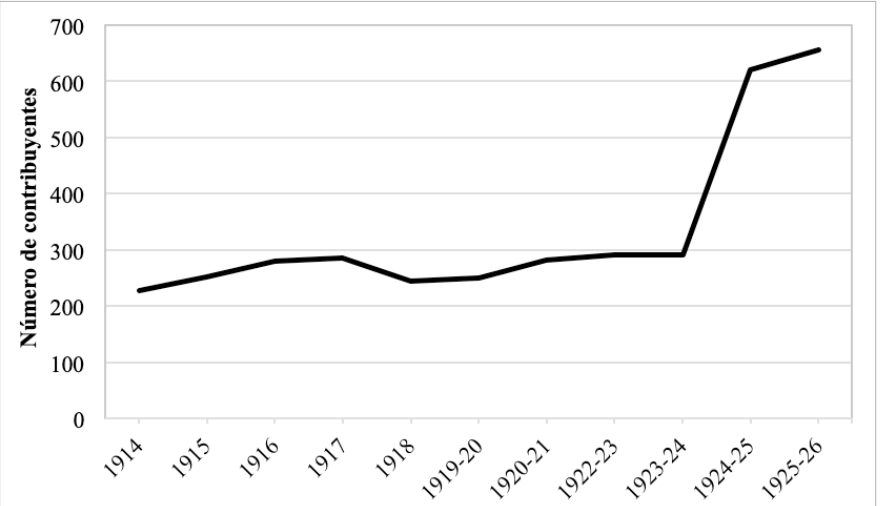

Figura 7. Número de matrículas de la contribución industrial y de comercio en Hellín, 1914-1926.

Fuente: Matrículas de la contribución industrial y de comercio de Hellín, AMH. Elaboración propia.

\footnotetext{
${ }^{11}$ Como resultado de la demanda excepcional de los países en guerra, en España el número de sociedades constituidas creció de forma significativa, pasando de 1272 en 1914 a 2143 en 1917, un crecimiento empresarial que se extendió hasta 1920, con 3085 sociedades creadas (Tafunell, 2005, p. 768). A pesar de ello, la especulación, la proliferación de pequeñas empresas y la insuficiente dotación de capital social dificultó la innovación tecnológica y la creación de una estructura industrial desarrollada y competitiva (Carreras y Tafunell, 2010; Comín, 2016).
}

En Hellín, como ocurrió en otras poblaciones de la España rural interior, los empresarios fabriles tuvieron una presencia reducida. Los avances fueron pausados, la diversificación económica lenta y reinó una marcada atonía tecnológica (Dobado y López, 2001; Pardo, 2000). Por ello, la agricultura seguía siendo en las primeras décadas del siglo xx la base fundamental de la economía local y la que marcaba, con la evolución de sus cosechas, el compás de la actividad económica (Cañabate, 2015, 2016). Para conocer qué efectos económicos pudo tener la epidemia de gripe de 1918 en el sector agrario, la figura 8 presenta los índices de producción de cereales, vino y aceite en la provincia de Albacete entre 1900 y 1930 . Considerada en conjunto, se observa que la producción agraria de los principales cultivos de la agricultura albaceteña aumentó progresivamente, algo explicable por el incremento relativo de los rendimientos de la tierra, pero también por la mejora en la productividad del trabajo, con el mayor empleo de abonos químicos y la mayor mecanización de ciertas labores agrícolas (Pardo, 2000; Valle Calzado, 2010). Las mayores tasas de crecimiento se dan entre 1912-1913 y 1915-1916, imputable en gran medida al aumento de la demanda externa por la Gran Guerra (Valle Calzado, 2010). A lo largo del período, no obstante, se advierten algunas coyunturas recesivas que alteran el ritmo de crecimiento agrario, como la de 1916-1919, con caídas pronunciadas en cereales, vino y aceite, y que deben ponerse en relación más con la retracción de la demanda exterior que tuvo lugar en la fase final de la guerra mundial que con un posible impacto de la pandemia, y el final de los años veinte, muy supeditado a la protección arancelaria y la caída de precios (Pardo, 2000; Valle Calzado, 2010).

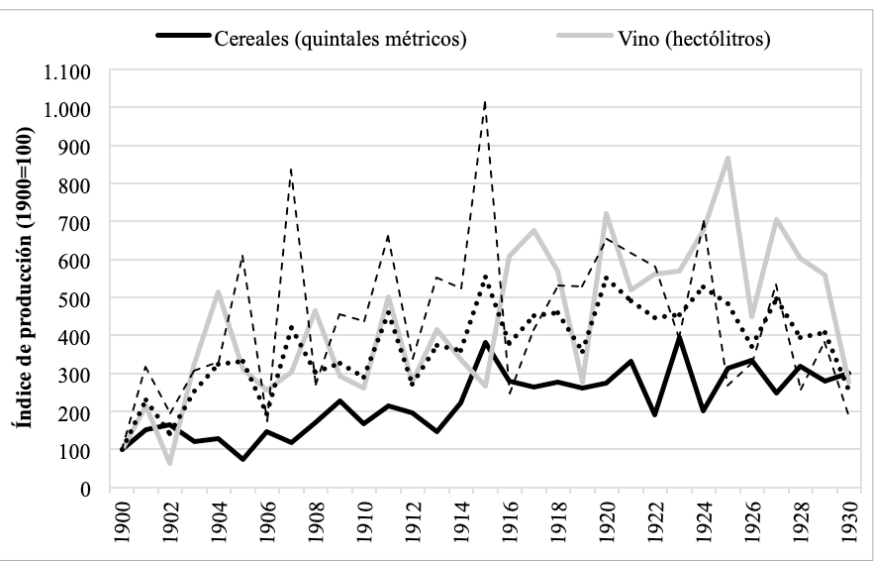

Figura 8. Índice de producción de cereales, vino y aceite en la provincia de Albacete, 1900-1930 (1900=100).

Fuente: Grupo de Estudios de Historia Rural (1991, pp. 117-138). Elaboración propia.

¿Cuál fue la evolución de los salarios en la zona? ¿Tuvo la gripe de 1918 efectos negativos sobre el nivel de vida de los trabajadores? Dada la ausencia de información salarial en Hellín, este trabajo ha utilizado como proxy los datos de los salarios nominales de los trabajadores agrícolas eventuales (hombres y mujeres) en el área vitivinícola del sureste español durante el primer tercio del siglo $\mathrm{xx}$, un área que incluye también la zona sureste de la provincia de Albacete (abarca los municipios que van desde Almansa a Hellín). Al tratarse de un espacio agrario muy homogéneo, tanto por el tipo de relacio- 
nes laborales y el mercado de trabajo como por las estructuras de la propiedad y explotación de la tierra (Martínez Soto, 2002), la utilización de los datos salariales de esta área como proxy del nivel de vida en Hellín se justifica para este período. La estadística salarial que se emplea es el resultado del estudio que Martínez Soto $(2002,2006)$ realizó a cuatro contabilidades privadas de distintas explotaciones agrícolas del área ${ }^{12}$, con condiciones climáticas y estructuras laboral-agrarias muy parecidas.

La figura 9 muestra, en general, una tendencia de crecimiento de los salarios nominales abonados a los trabajadores agrícolas eventuales en el área vitícola del sureste español entre 1900 y 1930 . Entre ambas fechas, los salarios de jornaleros y jornaleras crecieron 4,5 pesetas y 2,5 pesetas, respectivamente. Este incremento salarial, sin embargo, no fue sostenido al apreciarse un estancamiento durante los tres primeros lustros, e incluso un deterioro relativo entre 1901 y 1906, a causa del impacto de la filoxera en el viñedo en la zona (Piqueras, 2005; Valle Calzado, 2010). Por el contrario, a partir de 19171918 se advierte una subida significativa de los salarios, explicable, por una parte, por la presión que ejercieron los sindicatos de trabajadores agrícolas en los incipientes procesos de negociación colectiva a fin de compensar las fuertes alzas de los precios y, de otra, por la recuperación que experimentaron los precios del vino y la mejora de las redes de comercialización (Martínez Soto, 2002, pp. 225-256).

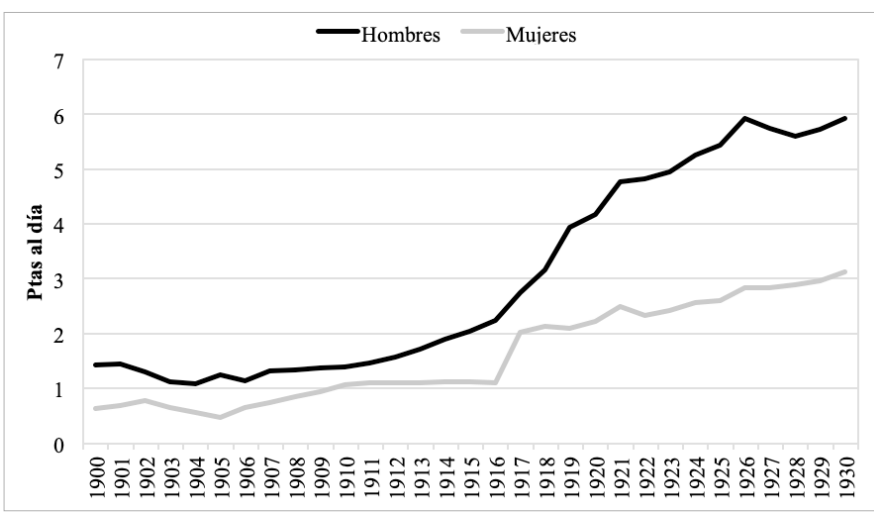

Figura 9. Evolución de los salarios nominales medios de los trabajadores agrícolas eventuales en el sureste español (comarcas del Altiplano, Valle de Vinalopó y Almansa) en las labores ordinarias*, 1900-1930 (pesetas al día).

* Labores ordinarias: cava, siembra, ahoyado, limpieza de cultivos y parcelas y abonado de tierras.

Fuente: Martínez Soto (2002, p. 653). Elaboración propia.

Los datos sugieren que la pandemia de gripe de 1918 apenas tuvo efectos negativos sobre el nivel de vida de las clases trabajadoras agrícolas del sureste español. Se constata, eso sí, una desigualdad salarial según género. Los salarios masculinos eran más altos siempre que los femeninos. Las diferencias fueron reducidas al inicio de siglo, aumentaron gradualmente en la década de 1910 y se agrandaron después de la Primera Guerra Mundial (figura 9). El aumento gradual que experimentaron los salarios nominales en el área vitícola del sureste es-

${ }^{12}$ Se trata de las explotaciones de Vidal Herrero-Albert y García CandelaIbáñez en el municipio de Yecla (Murcia); de Rico Payá en Villena (Alicante), y de Rubio Alcazar en Almansa (Albacete), todos ellos con una fuerte especialización en el cultivo del vino (Martínez Soto, 2002, p. 239). pañol, sin embargo, no se tradujo siempre en una mejora de los salarios reales, esto es, del poder adquisitivo para obtener bienes y servicios. Así se desprende de la figura 10, que muestra la evolución que siguieron entre 1909 y 1930 los salarios reales en la comarca del Altiplano (Murcia), próxima a Hellín, $\mathrm{y}$ el índice de precios al por menor de los artículos de primera necesidad en los pueblos de la región de Murcia.

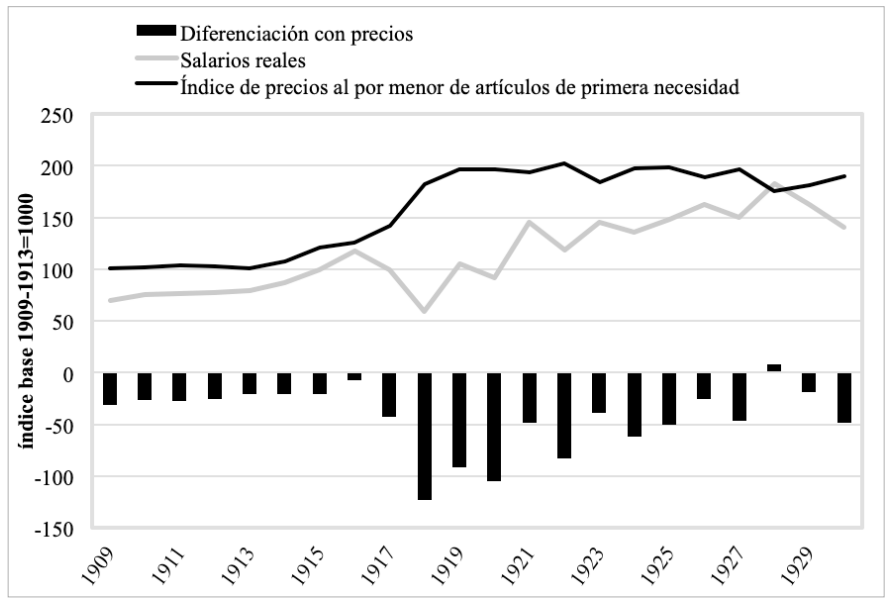

Figura 10. Evolución de los salarios reales en la comarca del Altiplano YeclaJumilla e índice de precios al por menor de los artículos de primera necesidad en los pueblos de la región de Murcia, 1909-1930.

Fuente: Martínez Soto (2006, p. 594). Elaboración propia.

Se aprecia que la fase final de la guerra mundial truncó súbitamente la tendencia alcista de los salarios reales que se había inaugurado en los años 1914-1916. No es que los salarios nominales no creciesen, que lo hicieron, pero no tanto como la inflación. Como resultado, el ingreso real de los trabajadores agrícolas del Altiplano murciano se erosionó, prolongándose hasta 1928. Hasta ese año, los salarios reales de los jornaleros de Yecla y Jumilla estuvieron por debajo del nivel de precios. Los períodos menos alejados de la inflación fueron los quinquenios 1912-1916 y 1926-1930, con una diferencia media de $-19,1$ y -26,3 puntos, respectivamente. Por el contrario, el período en el que se alcanzó el mayor diferencial con el nivel de precios fue el del quinquenio 1918-1922, con $-89,8$ puntos de media, consecuencia no de la pandemia de gripe de 1918, sino de la tendencia inflacionista que provocó la contienda mundial y que se prolongó durante los años inmediatos a la terminación de la misma (Martínez Soto, 1989).

La neutralidad de España en la Gran Guerra produjo efectos económicos beneficiosos. La necesidad de sustituir importaciones paralizadas por el conflicto y la expansión de las exportaciones a los países beligerantes inauguró un ciclo expansivo que benefició a algunos sectores de la economía nacional, aunque también perjudicó a otros, como los hortofrutícolas. La producción, los beneficios y el empleo crecieron, pero también los precios que desataron una espiral inflacionista (Carreras y Tafunell, 2010). La figura 10 revela que el índice de precios al por menor de los artículos de primera necesidad en los municipios de la región de Murcia se duplicó entre 1914 y 1919, manteniéndose elevado hasta finales de los años veinte. En contraste, las clases trabajadoras conocieron un empeoramiento de su nivel de vida, dado que la inflación no fue acompañada de un aumento equivalente en los salarios nominales. La brecha entre inflación y salario real se amplió a comienzos de 
los años veinte, disminuyendo gradualmente a partir de 192324 , gracias a la acción reivindicativa del sindicalismo jornalero en los incipientes procesos de negociación colectiva (Martínez Soto, 2006).

\section{Conclusiones}

La propagación y la mortandad ocasionadas por la pandemia del coronavirus han propiciado que se hayan rememorado los efectos de otras catástrofes epidémicas del pasado (Pujadas-Mora y Santiago-Caballero, 2020), entre las que sobresale la epidemia de gripe de 1918 (Ferreira, 2020; Mølbak Ingholt et al., 2020). La mayor crisis epidémica del siglo xx mató entre 1918 y 1920 a más de 50 millones de personas en el mundo, de los cuales alrededor de 260000 fallecieron en España, provocando, además, el mayor descenso vegetativo de la población en el siglo xx (Echevarri, 1993). Recogiendo este interés historiográfico, este artículo ha analizado los efectos de la gripe de 1918 en la morbimortalidad y en la economía de La Mancha meridional. Para ello, se ha estudiado el caso del municipio de Hellín, en la provincia de Albacete. Con una población media de 18290 habitantes en la década de 1910, Hellín era el segundo municipio más poblado de la provincia tras la capital, siendo representativo de La Mancha meridional.

Los resultados revelan que el descenso de la mortalidad y el proceso de modernización demográfica que Hellín había iniciado a comienzos del siglo xx se truncaron con la irrupción de la epidemia de gripe de 1918. La enfermedad epidémica provocó que la mortalidad se duplicara, al pasar de una tasa bruta del 23,2 por 1000 habitantes en 1917 a situarse en el 47,9\% en 1918. La mortalidad infantil en el primer año de vida, por su parte, se disparó alcanzando una tasa de muertes de casi 300 por 1000 nacidos vivos. Según nuestros cálculos, el resultado obtenido de aplicar el índice de Depâquier, con un valor de 8,3, apunta a que Hellín sufrió una crisis fuerte de mortalidad asociada a la pandemia de gripe. La mayor mortalidad epidémica provocó no solo una desaceleración en el ritmo de crecimiento de la población en el decenio de 1910, sino también un retroceso de la edad media de supervivencia de la población masculina, femenina y total hellinera, utilizada en este trabajo como proxy de la esperanza de vida. La consulta de otros estudios publicados muestra que, a diferencia de lo acontecido en otros contextos más urbanos (caso de Madrid), en Hellín la mayor letalidad se dio en la segunda oleada, en otoño de 1918, destacando también la mortalidad por enfermedades de tipo respiratorio y gastroenteritis. Probablemente, el hecho de que la primera oleada de gripe (primavera de 1918) tuviera poca incidencia en Hellín, pudo provocar que su población presentara un menor grado de inmunidad vírica en comparación con otras poblaciones más urbanizadas. Los datos, asimismo, muestran que existió una leve sobremortalidad masculina y que las edades más afectadas por la gripe fueron los menores de 4 años y la población comprendida entre los 10 y 39 años.

$\mathrm{Si}$ los efectos de la pandemia en la mortalidad fueron indiscutibles, su impacto en la economía local no fue tan perceptible. Los datos correspondientes a las matrículas de la contribución industrial y de comercio muestran que, aunque entre 1917 y 1918 se produjo un descenso relativo en el número de matriculaciones industriales y de comercio en Hellín, esta caída debe atribuirse sobre todo al final de la expansión económica y la crisis de los sectores que más crecieron durante el conflicto mundial, en nuestro caso el sector agroalimentario.
En el ámbito de la agricultura, la tendencia de los índices de producción de los principales cultivos en la provincia de Albacete (cereales, vino y aceite), aunque alcista a lo largo del primer tercio del siglo xx, presentó algunas coyunturas críticas, como la de 1916-1919, resultado también de la contracción que experimentó la demanda exterior en la fase final de la Gran Guerra. Durante estos años y la inmediata posguerra, el nivel de vida de los trabajadores agrícolas en el área vitivinícola del sureste español se deterioró. Y no porque los salarios nominales no aumentasen, que sí lo hicieron significativamente gracias al papel que desempeñaron los sindicatos agrícolas en los incipientes procesos de negociación colectiva, pero en menor medida que la inflación, en concreto respecto a los precios de los artículos de primera necesidad.

\section{Agradecimientos}

Este artículo se ha realizado en el marco de los proyectos de investigación PGC2018-095529-B-I00 (Ministerio de Ciencia, Innovación y Universidades), HAR2016-76814-C2-2-P (Ministerio de Economía y Competitividad), proyecto «Interreg-Sudoe VINCI-SOE3/P2/F0917» (FEDER-Unión Europea), del grupo de investigación S55_20R (Gobierno de Aragón y Fondo Europeo de Desarrollo Regional, «Construyendo Europa desde Aragón") y de la red de investigación RED2018-102413-T (Ministerio de Ciencia, Innovación y Universidades). Los autores agradecen las observaciones recibidas de los evaluadores anónimos.

\section{Bibliografía}

Almond, D. (2006). «Is the 1918 influenza pandemic over? Long-term effects of in utero influenza exposure in the post-1940 U.S. population", Journal of Political Economy, 114 (4), 672 -712.

Álvarez, P. E., Fernández López, A., González Taboada, F., Höfer, J. y Gómez Gómez, P. (2008). «Mortalidad en los concejos de Oviedo, Gijón y Avilés durante la epidemia de gripe de 1918», Revista miscelánea de Investigación, 22, 93-106.

Altares, G. (2020-05-10). "La memoria borrada de la gripe española», El País, Ideas, 6.

Ansart, S., Pellat C. y Boelle P. V. (2009). «Mortality Burden of the 1918-1919 Influenza Pandemic in Europe», Influenza and Other Respiratory Viruses, 3, 99106.

Barker, D. J. P. (ed.) (1992). Fetal and Infant Origins of Adult Disease. Londres: British Medical Journal.

Bell, F., y Millward, R. (1998). "Public health expenditures and mortality in England and Wales, 1870-1914», Continuity and Change, 12, p.221-249.

Beltrán. C. (1999). «La contribución industrial y el desarrollo industrial en España, 1845-1936» en De la Torre, J., y García-Zúñiga, M. (eds.) Hacienda y crecimiento económico: la reforma de Mon, 150 años después. Navarra: Gobierno de Navarra, pp. 143-168.

Bernabeu Mestre, J., Ramiro Fariñas, D., Sanz Gimeno, A. y Robles González, E. (2003). «El análisis histórico de la mortalidad por causas. Problemas y soluciones», Revista de Demografía Histórica, XII, 167-193.

Blanes, A. (2007). La mortalidad en la España del siglo xx. Análisis demográfico y territorial [tesis doctoral inédita]. Barcelona: Universidad Autónoma de Barcelona.

Camacho, J. (1999). La población de Castilla-La Mancha (siglos XIX y XX). Toledo: Junta de Castilla-La Mancha.

Cañabate, J. (2015). Estatura, salud y niveles de vida en Castilla-La Mancha. El caso de Hellín, 1887-2000 [tesis doctoral inédita]. Murcia: Universidad de Murcia.

Cañabate, J. (2016). «Niveles de vida biológico en Castilla-La Mancha durante el siglo xx. El caso de Hellín», Investigaciones de Historia Económica-Economic History Research, 12, 34-44.

Carreras, A. y Tafunell, X. (2010). Historia Económica de la España Contemporánea (1789-2009). Barcelona: Crítica.

Castelló, I. (2011). «Higiene alimentaria y mortalidad en la España contemporánea (1900-1974): el ejemplo de la fiebre tifoidea y la diarrea y enteritis en 
menores de dos años», Revista Española de Nutrición Humana y Dietética, 15 (2), 41-46.

Cilek, L., Chowell, G. y Ramiro Fariñas, D. (2018). «Age-Specific Excess Mortality Patterns During the 1918-1920 Influenza Pandemic in Madrid, Spain», Epidemiology in History, 187 (12), 2511-2523.

Chowell G, Erkoreka A, Viboud C. y Echeverri, B. (2014). «Spatial-temporal excess mortality patterns of the 1918-1919 influenza pandemic in Spain», BMC Infectious Diseases, 14, 371.

Comín, F. (2006). «Industrialización y desarrollo económico en la España contemporánea: una perspectiva histórica», en Gallego, G., Germán, L. y Pinilla, V. (eds.). Estudios sobre el desarrollo económico español. Zaragoza: Prensas de la Universidad de Zaragoza, pp. 43-67.

Correia, S., Luck, S. y Verner, E. (2020). «Pandemics Depress the Economy, Public Health Interventions Do Not: Evidence from the 1918 Flu». Disponible en: http://dx.doi.org/10.2139/ssrn.3561560.

Cutler, D. y Miller, G. (2005). «The Role of Public Health Improvements in Health Advances: The Twentieth-Century United States», Demography, 42, 1-22.

Dobado, R. y López, S. (2001). «Del vasto territorio y la escasez de hombres: la economía de Castilla-La Mancha en el largo plazo» en Germán, L., Llopis, E., Maluquer de Motes, J. y Zapata, S. (eds.). Historia económica regional de España, siglos XIX y Xx. Barcelona: Crítica, pp. 238-270.

Durán, A. M. (2020). «La pandemia de gripe en la provincia de Badajoz», en Ferreira, A. (coord.). A gripe espanhola de 1918. Guimaraes: Casa de Sarmento, Centro de Estudios de Patrimonio, pp. 277-314.

Echeverri, B. (1993). La gripe española. La pandemia de 1918-1919. Madrid: CIS.

Erkoreka, A. (2006). La pandemia de gripe española en el País Vasco (1918-1919). Leioa: Museo Vasco de Historia de la Medicina y de la Ciencia y Facultad de Medicina (UPV/EHU)

Erkoreka, A. (2010). «The Spanish influenza pandemic in occidental Europe (1918-1920) y victim age», Influenza and Other Respiratory Viruses, 4 (2), 8189.

Escudero, A. y Simón, H. J. (2003). «El bienestar en España: una perspectiva de largo plazo, 1850-1991», Revista de Historia Económica, 21 (3), 525-565.

Ferreira, A. (ed.) (2020). A gripe espanhola de 1918. Guimaraes: Casa de Sarmento, Centro de Estudios de Patrimonio.

Floud, R., Fogel, R. W., Harris, B. and Hong, S. C. (2011). The Changing Body: Health, Nutrition, and Human Development in the Western World Since 1700. Cambridge: Cambridge University Press.

Floud, R., Fogel, R. W., Harris, B. y Hong, S. C. (eds.) (2014). Health, mortality and the standard of living in Europe and North America since 1700, vol. I. Cheltenham: Edward Elgar Publishing.

García Ferrero, S. (2018). La gripe de 1889-1890 en Madrid [tesis doctoral]. Madrid: Universidad Complutense de Madrid.

Gómez Redondo, R. (1992). La mortalidad infantil española en el siglo xx. Madrid: Centro de Investigaciones Sociológicas; Siglo XXI.

Gozálvez, V. (2003). «Natalidad y mortalidad de la población valenciana (18581960)», Cuadernos de Geografía, 77/74, 277-302.

Grupo de Estudios de Historia Rural (GEHR) (1991). Estadísticas históricas de la producción agraria española, 1859-1935. Madrid: Ministerio de Agricultura, Pesca y Alimentación.

Hatton, T. J. (2014). «How have Europeans grown so tall?», Oxford Economic Papers, 66 (2), 349-372.

Martín, H. (2012). Las crónicas de Cádiz. Diario inédito de un relato apócrifo. Cádiz: Centro Andaluz de las Letras.

Martínez, M., y Barona, C. (1996). «Repercusiones de la epidemia de gripe de 1918 sobre la mortalidad de la ciudad de Valencia», en Barona, J. L. y J. Micó (eds.). Salut i malaltia en els municipis valencians. Valencia: Seminari d'Estudis sobre la Ciència, Universitat de Valencia, pp. 249-262.

Martínez Soto, A. P. (2002). «Salarios, sindicalismo y proceso de negociación en el área vitivinícola del sureste español, 1890-1936», en Martínez Carrión J. M. (ed.). El nivel de vida en la España rural, siglos XVIII-XX, Alicante: Publicaciones de la Universidad de Alicante, pp. 235-283.

Martínez Soto, A. P. (2006). «Mercado de trabajo, asociacionismo jornalero y condiciones de vida en el viñedo murciano: la comarca del Altiplano (18901936)», en Bolòs, J., Jarne, A. y Viñedo, E. (eds.). Condicions de vida al món rural. V Congrés sobre sistemas agraris, organització social i poder local. Lleida: Institut D’Estudis Ilerdens, pp. 577-598.

Mckeown, T. (1978). El crecimiento moderno de la población. Barcelona: Antoni Bosch.

Mitchell, B.R. (2007). International Historical Statistics. Europe, 1750-2005. New York: Palgrave Macmillan.

Mølbak Ingholt, M., Simonsen, L. y Van Wijhe, M. (2020). «Same place, different stories: The mortality burden of the 1918-1920 influenza pandemic in Denmark», Investigaciones de Historia Económica, Economic History Research, $16,49-67$.

Moll, I., Salas, P., Pujadas, J. M. y Canaleta, E. (2014). La lluita per la vida. Administració, medicina i reforma sanitària (1820-1923). Mallorca: El Gall Editor.

Nadal. J. (1987). "La industria fabril española en 1900. Una aproximación», en Nadal, J., Carreras, A. y Sudrià, C. (comps.). La economía española en el siglo XX, Barcelona: Ariel, pp. 23-61.

Nicolau, R. (2005). "Población, salud y actividad», en Carreras, A., y Tafunell, X (eds.). Estadísticas históricas de España: siglos XIX y XX. Bilbao: Fundación BBVA, pp. 77-154.

Pardo, M. R. (coord.) (2000). Historia económica de Castilla-La Mancha (siglos XVIXX). Ciudad Real: Biblioteca Añil.

Pérez Moreda, V., Reher, D. y Sanz Gimeno, A. (2015) La conquista de la salud. Mortalidad y modernidad en la España contemporánea. Madrid: Marcial Pons.

Piqueras, J. (2005). «La filoxera en España y su difusión espacial: 1878-1926», Cuadernos de Geografía, 77, 101-136.

Pujadas-Mora, J. M y Santiago-Caballero, C. (2020). «Special issue. Pandemics in History», Investigaciones de Historia Económica-Economic History Research. 16, $1-2$.

Ramírez, F. J. (2012) El hospital de la segunda Aguada (1793-1854) [tesis doctoral inédita]. Cádiz: Universidad de Cádiz.

Sanz Gimeno, A. y Ramiro Fariñas, D. (2002a). «Infancia, mortalidad y niveles de vida en la España interior. Siglos XIX y xx», en Martínez Carrión, J. M. (ed.). El nivel de vida en la España Rural. Siglos XVIII-XX. Alicante: Universidad de Alicante, pp. 359-402.

Sanz Gimeno, A. y Ramiro Fariñas, D. (2002b). «La caída de la mortalidad en la infancia en la España interior, 1860-1960: un análisis de las causas de muerte», Cuadernos de Historia Contemporánea, 24, 151-188.

Spinney, L. (2010) El jinete pálido. 1918: la epidemia que cambió el mundo. Barcelona: Crítica.

Szreter, S. (1988). "The importance of social intervention in Britain's mortality decline, 1850-1914: a reinterpretation of the role of public health", Social History of Medicine, 1, 1-38.

Szreter, S. (2020). «Rethinking McKeown: The relationship between public health and social change», American Journal of Public Health, 92 (5), 722-725.

Valle Calzado, A. R. (2010) Historia agraria de Castilla-La Mancha, siglos XIX-XX Ciudad Real: Almud Ediciones.

Tafunell, X. (2005). «Empresa y bolsa», en Carreras, A. y Tafunell, X. (eds.), Estadísticas históricas de España. Barcelona: FBBVA, pp. 707-833. 